\title{
Comparison of the Five Different Methods in Arterial Diameter Measurement
}

\author{
Orhan Babuccu ${ }^{1}$, Bulent Tekerekoglu ${ }^{1}$, Huseyin Ozdemir ${ }^{2}$, Halit Besir ${ }^{2}$, Sadi Gundogdu ${ }^{2}$ \\ ${ }^{1}$ Zonguldak Karaelmas University, Faculty of Medicine, Department of Plastic, \\ Reconstructive and Aesthetic Surgery, Zonguldak, Turkey \\ ${ }^{2}$ Zonguldak Karaelmas University, Faculty of Medicine, Department of Radiology, \\ Zonguldak, Turkey \\ E-mail: orhanbabuccu@karaelmas.edu.tr
}

Received January 21, 2011; revised May 6, 2011; accepted May 16, 2011.

\begin{abstract}
Background/Aims: In this study, the different types of arterial diameter measurement methods were compared with each other on rat model. Methods: The study was planned in three phases. In phase 1, all subjects $(n=30)$ underwent high resolution B-mode ultrasound examination and external diameter of the right common carotid artery (RCCA) was measured. In phase 2, RCCA was explored. In phase 3, rats were put into three groups. Group 1 was kept at $-20^{\circ} \mathrm{C}$, group 2 was embedded in $4 \%$ formaldehyde solution. In group 3 , circulatory system was filled with \%20 latex solution. In all groups, digital image of the RCCA was taken after the processes mentioned above. Images were assessed by Image-Pro Plus software. Results: The greatest average diameter was observed in the high resolution B-mode ultrasound (phase 1). In group 1 and 2, direct measurement of the RCCA on living animal (phase 2) gave significantly wider diameter than those obtained at phase 3 in the same groups. Direct (phase 2) and latex (phase 3) measurements were equal in group 3. Conclusions: High resolution B-mode ultrasound gives larger, probably actual diameter of the artery. Latex injection results might correspond those encountered during operation.
\end{abstract}

Keywords: Animal Model, Arterial Diameter, B-Mode Ultrasound

\section{Introduction}

When diameter of any artery is in concern, referring to textbooks and literature mostly reveals different values [1-3]. There could be several reasons to explain this inconsistency, such as age and sex of the subjects, external and internal factors influencing arterial diameter, viability of the subject (cadaver or living person), and finally measurement methods, which could be invasive or non invasive [4,5]. In this study, with keeping characteristics of the measured population (i.e. in this study rats' weight, age, and sex) constant, invasive procedures in both living subject and cadaver were compared with non-invasive method, which was high resolution B-mode ultrasound. It is a common and practical method to evaluate arterial diameter in vivo [6]. In this study accuracy high resolution B-mode ultrasound was compared with invasive measurements in both living and dead form of the same subject. As far as we concern, there is no other study using this type of methodology.

\section{Material and Methods}

Thirty male Wistar rats, weighing 200 to 250 g, were used. Animals were housed in plastic cages without restraint and fed standard rat chow, and given water ad libitum. No food restriction was given before anesthesia. All animals used in this study received human care under the supervision of Animal Research Ethic Comity of Zonguldak Karaelmas University, Faculty of Medicine.

\subsection{Experimental Groups}

Anesthesia was induced with intraperitoneal injection of pentobarbital $(40 \mathrm{mg} / \mathrm{kg})$. Of the all thirty animals, external diameter of the right common carotid artery (RCCA) was measured with transcutaneous high resolution B-mode ultrasound, while they were under anesthesia (phase 1). Once this procedure had been completed, the same artery was surgically explored under magnification (OM-5 operating microscope, Tagaki Seiko Co., 
Ltd., Japan, with three magnification level: 4.6×, 7.7×, $12.3 \times$ ) and picture of this region was taken as the animal still alive but under anesthesia (phase 2). After high resolution B-mode ultrasound examination and taking picture of the artery, animals were divided in to three groups randomly so that each group was consisted of ten animals (phase 3). In group 1, animals were sacrified with $100 \mathrm{mg} / \mathrm{kg}$ intraperitoneal pentobarbital and put in to the freezer. In group 2, after euthanasia, animals were fixed by immersion in $4 \%$ formaldehyde solution. Finally, following euthanasia, circulatory system of animals in group 3 were filled with $20 \%$ latex solution (Table 1).

\subsection{High Resolution B-Mode Ultrasound Protocol (Groups 1, 2, and 3)}

After right side of their neck was shaved, the rats underwent an ultrasonographic examination with B-mode ultrasound (ATL 5000 HDI, Bothell, USA) with a high-resolution, 5-12 MHz linear transducer. The external diameter of the RCCA was measured over a distance of about $1 \mathrm{~cm}$ proximal to the carotid artery bifurcation on supine position. To obtain reliable values, three consecutive measurements were made and average value were taken (Figure 1).

\subsection{In vivo Measurement (Groups 1,2, and 3)}

After ultrasound examination, animal was taken to operation plate in supine position and then, right side of its neck was incised down to reach RCCA. Keeping the temperature constant $\left(23-25^{\circ} \mathrm{C}\right)$ and the surgical area soaked with warm saline, animals were left to rest for ten minutes to reduce probable vasoconstriction due to surgical procedure. Eventually, the milimetric scale was put under artery and digital images of common carotid artery were taken with Nikon E995 digital camera, which was mounted to the microscope (Figure 2).

\subsection{Freezing (Group 1)}

This group is supposed to correspond to fresh cadaver studies. In a routine practice, it is almost impossible legally to study an anatomy on the man, who died recently. Generally, the corpus is kept in the freezer and thawed before the dissection. For this reason, after in vivo measurement (phase 2), the animal underwent euthanasia and put in to freezer within the nylon bag. The corpus was kept three days at $-20^{\circ} \mathrm{C}$ and at the end of this time, it was thawed at the room temperature. Finally, RCCA explored surgically and digital images were take under magnification as it was described above.

\subsection{Formaldehyde (Group 2)}

This group standed for the measurements made on the formalin fixed cadavers. At the end of the phase 2, the rats in this group were sacrificed and its abdominal wall was opened to explore abdominal aorta, which was cannulated with 24 gauge intravenous catheter. Subsequently, the blood in the circulatory system of rat taken out by the help of syringe as much as possible, 3 - 4 cc on average (phase 3). With the same catheter, 10 cc $10 \%$ formaldehyde was infused into circulatory system and aorta was tied to prevent oozing back. The animal was hold sank in $4 \%$ formaldehyde for one week. After this period, rat was taken out from solution, kept in the room temperature for six hours for aeration. Finally, RCCA explored surgically and digital images were taken as describe above.

\subsection{Latex (Group 3)}

Phase 1, and phase 2 were achieved as described previously. In this group, instead of formaldehyde, 10 cc $20 \%$ latex solution was infused under constant pressure and aorta was tied to prevent backward oozing. The animal was kept 24 hours in the refrigerator at $4^{\circ} \mathrm{C}$ to get latex harden. The final step was digital imaging of the RCCA.

\subsection{Measurement}

RCCA diameter at the point that $1 \mathrm{~cm}$ proximal to the bifurcation was gauged with Image Pro software (ImagePro Plus, 2000 version 4.5 for Windows) (Figure 3).

\subsection{Statistical Methods}

The correlation between measurements in groups was evaluated with Friedman Test. Wilcoxon Signed Ranks Test was used for further evaluation. For statistical significance, alpha level was presumed as 0.05 .

\section{Results}

The values from each subject were given in table (Table 2). Among four measurement methods, which were described above, the greatest average diameter was observed in the high resolution B-mode ultrasound examination in all groups (Group 1: $1.47 \mathrm{~mm} \pm 0,09 \mathrm{SD}$; Group 2: $1.38 \mathrm{~mm} \pm 0.11 \mathrm{SD}$; and Group 3: $1.39 \mathrm{~mm} \pm$ $0.11 \mathrm{SD})(\mathrm{p}=0.001)$. In group 1 and group 2 , in vivo average measurements $(1.24 \mathrm{~mm} \pm 0.13 \mathrm{SD}$ and 1.17 $\mathrm{mm} \pm 0.09 \mathrm{SD}$, respectively) were significantly greater than those measured in frozen $(1.09 \mathrm{~mm} \pm 0.10 \mathrm{SD}$; $\mathrm{p}=$ $0.001)$ and formaldehyde treated animals (1.07 $\mathrm{mm} \pm$ 
Table 1. The design of the study groups is presented.

\begin{tabular}{cccccc}
\hline & Doppler & In vivo imaging & Freezing & Formaldehyde & Latex \\
\hline Group I $(n=10)$ & + & + & + & - & - \\
Group II $(n=10)$ & + & + & - & + & - \\
Group III $(n=10)$ & + & + & - & - & + \\
\hline
\end{tabular}

$\square$ Phase 1, $\square$ Phase 2, $\square$ Phase 3.

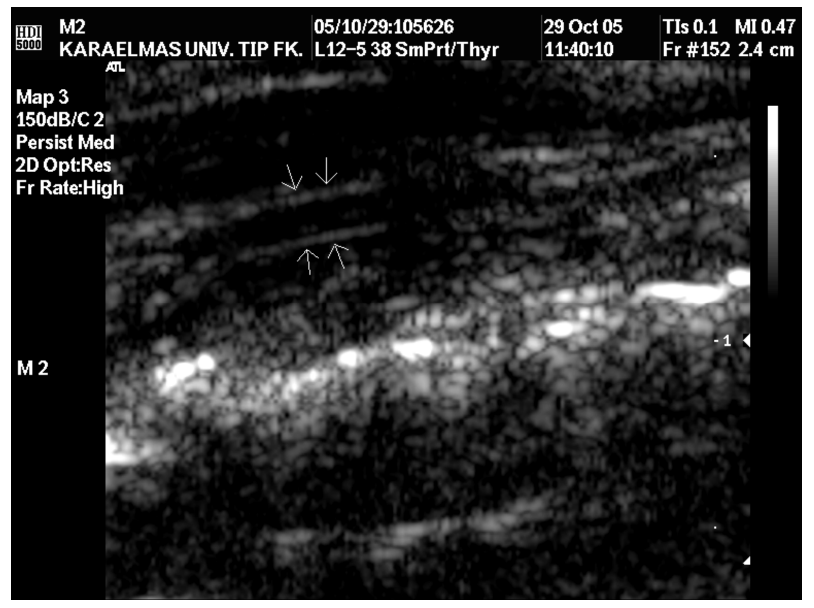

Figure 1. The image of the RCCA obtained by B-mode ultrasound. The external diameter was measured.

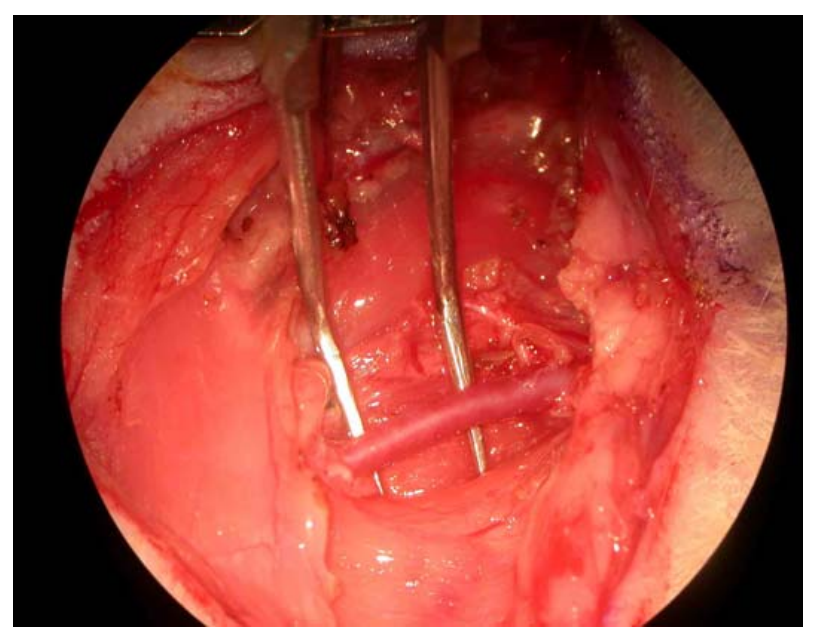

Figure 2. The picture was taken while subject was still alive (phase 2). After imaging, all subjects were euthanized, and phase 3 was started.

$0.08 \mathrm{SD} ; \mathrm{p}=0.001$ ), respectively. In group 3 , the average diameter measured in vivo and after latex fixation was the same, which was $1.27 \mathrm{~mm} \pm 0.07 \mathrm{SD}$ (Table 2, Figure 4).

\section{Discussion}

In vivo measurement techniques of the arterial diameter

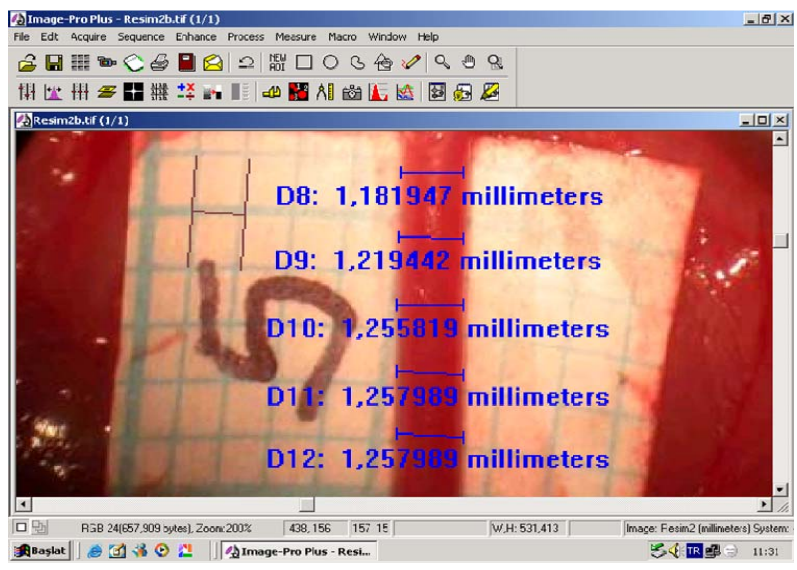

Figure 3. The measurements were done at five different levels on the image by Image Pro software (Image - Pro Plus, 2000 version 4.5 for Windows), and average value was taken as a diameter.

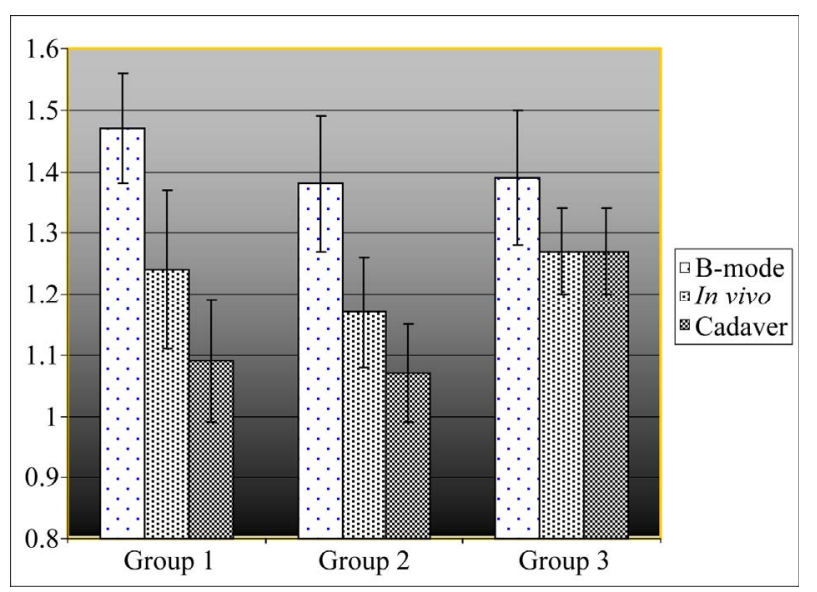

Figure 4. The average values obtained from each groups were presented in diagram.

are numerous. They may be indirect as in the radiological methods such as MR [7], CT [8] or ultrasound [9,10], or direct, using digital or manual calipers [11] during operation. In a cadaver, the arterial diameter can be either measured directly $[12,13]$ or after filling the arterial system with either of silicone [14], latex [15,16], or oxide-gelatin $[15,17]$.

In this study, we tried to compare different methods in the same artery, i.e. high resolution B-mode ultrasound, 
Table 2. The raw data obtained from each subject is presented.

\begin{tabular}{|c|c|c|c|c|}
\hline & Subjects & Doppler (mm) & In vivo $(\mathrm{mm})$ & Frozen (mm) \\
\hline & FR 1 & 1.15 & 1.60 & 0.90 \\
\hline & FR 2 & 1.34 & 1.60 & 1.06 \\
\hline & FR 3 & 1.04 & 1.40 & 1.05 \\
\hline & FR 4 & 1.29 & 1.50 & 1.12 \\
\hline & FR 5 & 1.45 & 1.70 & 1.24 \\
\hline \multirow[t]{12}{*}{ GROUP 1} & FR 6 & 1.39 & 1.70 & 1.26 \\
\hline & FR 7 & 1.13 & 1.60 & 1.08 \\
\hline & FR 8 & 1.12 & 1.50 & 1.03 \\
\hline & FR 9 & 1.27 & 1.60 & 1.17 \\
\hline & FR 10 & 1.22 & 1.50 & 1.07 \\
\hline & Average & $1.47 \mathrm{~mm} \pm 0.09 \mathrm{SD}$ & $1.24 \mathrm{~mm} \pm 0.13 \mathrm{SD}$ & $1.09 \mathrm{~mm} \pm 0.10 \mathrm{SD}$ \\
\hline & & & & Formaldehyde (mm) \\
\hline & $\mathrm{F} 1$ & 1.18 & 1.50 & 1.07 \\
\hline & F2 & 1.12 & 1.60 & 1.02 \\
\hline & F3 & 1.12 & 1.60 & 1.00 \\
\hline & F4 & 1.20 & 1.70 & 1.09 \\
\hline & F5 & 1.25 & 1.60 & 1.15 \\
\hline \multirow[t]{5}{*}{ GROUP 2} & F6 & 1.36 & 1.60 & 1.27 \\
\hline & F7 & 1.19 & 1.80 & 1.06 \\
\hline & F8 & 1.10 & 1.40 & 0.99 \\
\hline & F9 & 1.08 & 1.50 & 1.02 \\
\hline & F10 & 1.09 & 1.50 & 1.02 \\
\hline
\end{tabular}

\begin{tabular}{|c|c|c|c|c|}
\hline & Average & $1.38 \mathrm{~mm} \pm 0.11 \mathrm{SD}$ & $1.17 \mathrm{~mm} \pm 0.09 \mathrm{SD}$ & $1.07 \mathrm{~mm} \pm 0.08 \mathrm{SD}$ \\
\hline \multirow{12}{*}{ GROUP 3} & & & & Latex (mm) \\
\hline & L1 & 1.27 & 1.40 & 1.30 \\
\hline & L2 & 1.31 & 1.40 & 1.26 \\
\hline & L3 & 1.33 & 1.60 & 1.31 \\
\hline & L4 & 1.36 & 1.60 & 1.39 \\
\hline & L5 & 1.14 & 1.40 & 1.15 \\
\hline & L6 & 1.19 & 1.40 & 1.18 \\
\hline & L7 & 1.24 & 1.60 & 1.23 \\
\hline & L8 & 1.30 & 1.50 & 1.29 \\
\hline & L9 & 1.24 & 1.60 & 1.22 \\
\hline & L10 & 1.38 & 1.70 & 1.37 \\
\hline & Average & $1.39 \mathrm{~mm} \pm 0.11 \mathrm{SD}$ & $1.27 \mathrm{~mm} \pm 0.07 \mathrm{SD}$ & $1.27 \mathrm{~mm} \pm 0.07 \mathrm{SD}$ \\
\hline
\end{tabular}


direct in vivo measurement, and cadaver study of the right common carotid artery of the same rat. Among three different methods applied to the same artery, the widest diameter was found in high resolution B-mode ultrasound examination in all groups (Table 2, Figure 4). It was probably actual diameter of the given artery in living subject. In group 1 and 2, the values obtained during phase 2 (in vivo measurement) were significantly grater than those obtained from frozen and formalin fixed cadavers. In contrast, the average diameter directly measured on living subject and that measured on latex filled artery within the same subject were equal. In fact, this is not unexpected, since latex can support vessel wall as blood pressure does.

\section{Conclusions}

1) Unless filled immediately with latex or silicon, vascular diameter values measured in cadaver will be lesser than in vivo values.

2) In living person, high resolution B-mode ultrasound is practical way to measure arterial diameter. On the other hand, the results probably will always be greater than those written classical textbooks.

\section{References}

[1] B. Strauch and H. L. Yu, "Atlas of Microvascular Surgery: Anatomy and Operative Approaches," Thieme Medical publishers, Inc., New York, 1993.

[2] S. J. Mathes and F. Nahai, "Reconstructive Surgery: Principles, Anatomy and Technique," Churchill Livingstone, Edinburgh, 1997.

[3] D. Serafin, "Atlas of Microsurgical Composite Tissue Transplantation,” Saunders Company, Philadelphia, 1996.

[4] O. Babuccu, H. Ozdemir, M. Hosnuter, E. Kargi, A. Sogut and F. N. Ayoglu, "Cross-Sectional Internal Diameters of Radial, Thoracodorsal, and Dorsalis Pedis Arteries in Children: Relationship to Subject Sex, Age, and Body Size,” Journal of Reconstructive Microsurgery, Vol. 22, No. 1, 2006, pp. 49-52. doi:10.1055/s-2006-931907

[5] I. H. Peterson, R. E. Jensen, J. Parnell, "Mechanical Properties of Arteries In vivo," Circulation Research, Vol. 8, 1960, pp. 622-639.

[6] A. Munk, K. Darge, M. Wiesel, J. Troeger, "Diameter of the infrarenal aorta and the iliac arteries in children: ultrasound measurements," Transplantation, Vol. 73, No. 4, 2002, pp. 631-635. doi:10.1097/00007890-200202270-00028

[7] H. C. Assen, A. de Roos, J. Vanderschoot and J. H.
Reiber, "Vessel Diameter Measurements in Gadolinium Contrast-Enhanced Three-Dimensional Mra of Peripheral Arteries,” Magnetic Resonance Imaging, Vol. 18, No. 1, 2000, pp. 13-22. doi:10.1016/S0730-725X(99)00099-5

[8] S. Suzuki, S. Furui, T. Kaminaga and T. Yamauchi, "Measurement of Vascular Diameter In vitro by Automated Software for Ct Angiography: Effects of Inner Diameter, Density of Contrast Medium, and Convolution Kernel,” American Journal of Roentgenol, Vol. 182, No. 5, 2004, pp.1313-1317.

[9] R. W. Stadler, W. C. Karl and R. S. Lees, "New Methods for Arterial Diameter Measurement from B-Mode Images," Ultrasound in Medicine and Biology, Vol.22, No.1, 1996, pp. 25-34. doi:10.1016/0301-5629(95)02017-9

[10] V. R. Newey, D. K. Nassiri, “Online Artery Diameter Measurement in Ultrasound Images using Artificial Neural Networks," Ultrasound in Medicine and Biology, Vol. 28, No. 2, 2002, pp. 209-216. doi:10.1016/S0301-5629(01)00505-1

[11] A. Uehata, T. Matsuguchi, J. A. Bittl, et al, “Accuracy of Electronic Digital Calipers Compared with Quantitative Angiography in Measuring Coronary Arterial Diameter," Circulation, Vol. 88, No.4 Pt 1, 1993, pp.1724-1729.

[12] G. D. Rosson, L. H. Holton, R. P. Silverman, N. K. Singh, M. Y. Nahabedian, "Internal Mammary Perforators: A Cadaver Study,” Journal of Reconstructive Microsurgery, Vol. 21, No. 4, 2005, pp. 239-242. doi:10.1055/s-2005-871750

[13] I. K. Lukic, V. Gluncic, A. Marusic, "Extracranial Braches of the Middle Meningeal Artery," Clinical anatomy, Vol. 14, No. 4, 2001, pp. 292-294. doi:10.1002/ca.1051

[14] O. Magden, M. Edizer, A. Atabey, V. Tayfur, I. Ergur, "Cadaveric Study of the Arterial Anatomy of the Upper Lip,” Plastic and Reconstructive Surgery, Vol. 114, No. 7, 2004, pp. 355-359. doi:10.1097/01.PRS.0000131876.45116.77

[15] K. Hwang, W. J. Lee, C. Y. Jung, I. H. Chung, “Cutaneous Perforators of the Upper Arm and Clinical Applications," Journal of Reconstructive Microsurgery, Vol. 21, No. 7, 2005, pp.463-469. doi:10.1055/s-2005-918901

[16] K. Z. Tao, E. Y. Chen, R. M. Ji and R. S. Dang, “Anatomical Study on Arteries of Fasciae in the Forearm Fasciocutaneous Flap,” Clinical Anatomy, Vol. 13, No. 1, 2000, pp.1-5. doi:10.1002/(SICI)1098-2353(2000)13:1<1::AID-CA1>3 $.0 . \mathrm{CO} ; 2-6$

[17] B. P. Thomas, C. R. Geddes, M. Tang, J. Williams and S. F. Morris, "The Vascular Basis of the Thoracodorsal Artery Perforator Flap,” Plastic and Reconstructive Surgery, Vol. 116, No. 3, 2000, pp. 818-822. doi:10.1097/01.prs.0000176253.42394.7c 